\title{
Summarize of Technological Development about Integrated Simulation of Gas Turbine Equipment
}

\author{
LI Shu-ying, LI Tie-lei, WANG Zhi-tao \\ College of Power and Energy Engineering \\ Harbin Engineering University, HEU \\ Harbin, China \\ e-mail: lishuying@hrbeu.edu.cn, 303730585@qq.com, wangzhitao@hrbeu.edu.cn
}

\begin{abstract}
This paper summarizes integrated simulation technology development status of gas turbine equipment at home and abroad, makes comparative analysis of the deficiencies in the existing domestic research, forecasts the direction of development in the field of integrated simulation about gas turbine equipment at our country finally.
\end{abstract}

Keywords-gas turbine equipment; system simulation; integrated simulation; summarize

\section{INTRODUCTION}

The development of high-performance gas turbine equipment epitomizes the strength of a country's industry and defense. The traditional gas turbine equipment development process to physical prototype trial and test assessment results as the foundation, not only has high cost and long cycle, and put into the compressor surge margin, causing unit scrapped great risk. The introduction of system simulation technology can form a "simulation driven development process for gas turbine equipment" (As shown in Figure 1). The comparision of two kinds of development process can be seen, the latter has obvious advantages in reducing test risks, save development costs and shortening development cycle.

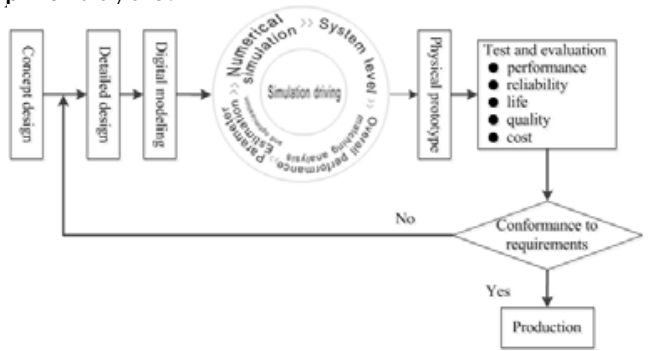

Figure 1. Driving driving gas turbine equipment development process.

The concept of integrated simulation is proposed on the basis of system simulation technology gradually mature. In 1984 Orën propose that simulation activity had three sections, which are "modeling-simulation-analysis of simulation results". Based on the above understanding, J. O. Henriksen, who first propose the concept of integrated simulation environment [1]. First, the integrated simulation environment is a combination of software tools, support simulation activity to complete all the works. Second, the integrated simulation environment with unified man-machine interface and operating style, can achieve data sharing in the whole process of simulation, through the database technology, it greatly improves the efficiency of simulation activity. As the simulation objects become more complicated, the time and labor cost of simulation activity rising, "simulation is a model-based activity", so the integrated simulation must give sufficient support to the reuse of simulation models, in order to reduce the cost of complex system simulation activity. Specific to the integrated simulation of gas turbine equipment, supporting simulation model reuse includes two meanings: one is to support the reuse and coupling of component simulation models with different dimensions, achieving system simulation zoom; the second is to support the reuse and coupling of component simulation models involving different disciplines, to support the multilevel and multidisciplinary nature of gas turbine equipment development process.

\section{GAS TURBINE EQUIPMENT MODULAR INTEGRATED SIMULATION TECHNOLOGY}

\section{A. Modular Integrated Simulation Technology Overview}

Compared with holistic modeling, modular modeling can reduce the difficulties of system analysis and greatly promote the reuse of simulation models. Although the application form and working fluid circulation mode have a great change in the span, from stand-alone to combined power plant, from uniaxial simple cycle to multiaxial complex cycle, but the overall system can be regarded as a few class components whose functions are independent each other, the working fluid movement process must abide by the mass, energy and momentum conservation relationship, the working fluid state parameters must also meet the function relations between thermal properties. Based on the above analysis, we can establish all the component simulation models for gas turbine equipment, using modular modeling method.

Gas turbine equipment modular modeling by reasonable modular to decompose, to establish modular model library, to establish public repository library, to design module operation aid and to develop simulation model and so on several aspects of content.

By using of high-level program language and its compiler environment or commercial simulation software, the mathematical model of module can be translated to simulation model, which is performed by computer. Modular integrated simulation technology to modular modeling as the 
foundation. The simulation model is by modular model library unified management, including the system modeling, simulation and the simulation results analysis, all operations are integrated in the unity of the environment, with database technology realize data sharing of simulation process, so as to better improve the simulation efficiency.

\section{B. Modular Integrated Simulation Research Status}

Early gas turbine equipment modular integrated simulation platform development is the use of processoriented high-level programming language and its compiler environment. Since the 1990s, object-oriented modeling technology by virtue of its inheritance, reusability and scalability characteristics gradually replace the traditional process-oriented modeling technology. The late 1990s, U.S. government launch a numerical simulation of a NASA leadership, a number of government departments, research institutions, enterprises and universities to participate in the propulsion system (NPSS, Numerical Propulsion System Simulation) plan [2]. The goal of the plan is using objectoriented technology, the establishment of a modular aircraft propulsion system virtual experiment platform, completion of the full size aircraft engine in detail simulation. NPSS plan mainly include engine application model, integrated simulation environment and high performance computing environment three aspects. Which can be refined engine application model for component integration, multidisciplinary coupled simulation and simulation zoom three sub-sections. NPSS integrated simulation environment is based on an object-oriented technology development on 0D module library. Different researchers can operate these modules include completion of multidisciplinary analysis, simulation zoom, data management, the main function of NPSS. In fact belong to different discipline, with different dimension (from 1-D to 3-D) component models are through the distributed simulation interface and module library of 0$\mathrm{D}$ module coupling and unified managemen.

The United States UTC company to carry out ITAPS project [3], by using the object-oriented technique develop a generic business aircraft engine simulation system. Through standard interfaces, the component models which belong to different discipline, are integrated into a unified simulation framework to solve the simulation models reuse.

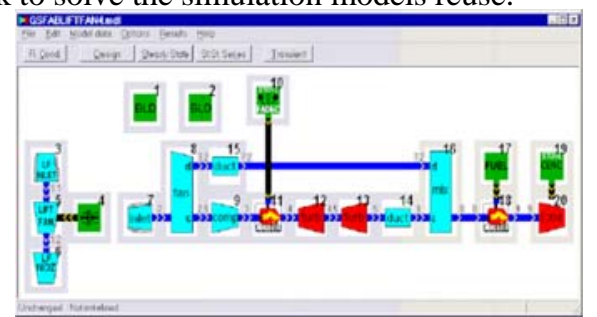

Figure 2. The GSP operating enviroment.

The gas turbine performance simulation software GSP also has a modular operating environment [4] (as shown in Figure 2), which is developed by Holland National Aircraft Laboratory, based on object-oriented environment Borland Delphi. GSP integrat extensible component model library and control system library, simulation can be of many types of aircraft engine with control system.

Xie Zhiwu of Shanghai Jiao Tong University do a lot of research on object-oriented analysis and design, construct a object-oriented class framework [5], which searve on the gas turbine system of arbitrary structure and simple cycle, the various computing tasks (steady simulation and dynamic simulation) for gas turbine simulation are summarized as component calculation, flow path calculation and and system state calculation three levels, and put forward that component should be calculated using the "node-connectorcomponent" model structure, so that the component model, working fluid flow and simulation algorithm can be extended. On this basis, the source codes are developed by using of $\mathrm{C}++$ language. Finally, the validity of framework and expanded capacity are verified, using a three-shaft gas turbine cubage inertia simulation. The reference [6] combining with traditional waterfall model in software engineering theory, develop a line with gas turbine simulation characteristics of software engineering implementation program (SPGTS, Software Process of Gas Turbine Simulation), in order to ensure that in gas turbine simulation software development process, all the elements can be effectively managed; on this basi, using unified modeling language designs a general system object-oriented class framework Vite, which is conducted on the static description, using extensible markup language and mathematical markup language, completes the development for domain model of SPGTS; finally, according to the SPGTS standard, a heavy duty gas turbine integrated simulation platform is developed, using of C\# language.

Making use of general commercial simulation software can also develop gas turbine equipment modular integrated simulation platform. GASTURBOLIB [7] is a simple cycle gas turbine $0-\mathrm{D}$ performance simulation software, which is developed by Siemens Company, based on SIMULINK environment, gas turbine component models, including all types of controller, are packaged in model library as modules for unified management. Sweden LUND college and the Italy Air Force Research Institute to launch MOGM project [8], based on the development environment of Dymola/Modelica develop gas turbine system simulation platform, the platform as a general, scalable modular model library based on, can complete system performance analysis for gas turbine with various structures. The reference [9] in Microsoft Excel environment using VB language develop a turbojet visualization dynamic simulation program, and discuss the use of Excel broad compatibility with the possibility of simulation software coupling integration.

Cao Yuan of Shanghai Jiao Tong University [10] based on Dymola/Modelica environment develop aircraft engine nonlinear modular model library, which include components, modules and class such as three levels. The component level is partitioned according to aircraft engine physical entity; the module level according to the working mechanism is divided into mechanical, flow, volume, medium, interface modules of the five types; class is smallest unit of the model library, its main function is to carry out calculation of thermodynamics parameters. Study of reference [11] based 
on the EASY 5 development of the marine gas turbine modular model library, through simulation and experiment proves that this model has good expansibility and real-time simulation capability.

\section{GAS TURBINE EQUIPMENT INTEGRATED SIMULATION TECHNOLOGY WITH COUPLED SOFTWARES}

\section{A. Based on Interface of Integrated Simulation Technology with Coupled Softwares Overview}

In the modular modeling process, we can develop models, making full use of a variety of different characteristics of commercial simulation softwares, to accelerate the speed of modeling. Usually, each kind of commercial simulation software is specific discipline, supporting a particular dimension simulation model. If the system simulation can be completed, by use of coupleing component or subsystem simulation models, which are developed by different simulation softwares, not only can play different simulation softwares to their respective advantages, to meet the different simulation requirements of gas turbine research, but also can effectively promote the reuse of simulation models. The functions of interface are to guarantee the data communication between different simulation softwares and simulation synchronization, which are the key of achieving softwares coupling.

\section{B. Integrated Simulation with Coupled Softwares Research Status}

Developed countries have developed expandable gas turbine equipment integrated simulation platform, which has the ability of simulation zoom, multidisciplinary coupling analysis and distributed simulation, so they don't require the aid of coupling general simulation softwares to conduct research.

Harbin Institute of Technology, Bowen, Li Weipeng et al [12] achieve the synchronization of FLUENT and SIMULINK two simulation softwares, using the he blocking technology in TCP/IP communication protocol, on this basis, develop interface and establish a scramjet distribution parameter closed loop control simulation platform. The reference [13] establish by SIMULINK aircraft engine digital controller model, and through the S-Function interface establish the AMESim hydraulic actuator and C language engine ontology model coupling, to establish a set of aircraft engine control system simulation model. On this basis, a integrated simulation platform of aircraft engine control system is developed, which includes model layer, data layer and the application laye, making use of VB language and Active X automation technology.

\section{GAS TURBINE EQUIPMENT DISTRIBUTED INTEGRATED SIMULATION TECHNOLOGY}

\section{A. Distributed Integrated Simulation Technology Overview}

Distributed simulation means to use coordinated structure, standard, protocol and database, through the network will be dispersed in different operation nodes the autonomy simulation models, form interactive integrated simulation environment. The autonomy of the simulation model means researchers in modeling and simulation process, according to the actual situation to choose a different development environment, running environment and the seek truth algorithm, simulation step length, this undoubtedly enhance the flexibility in the process of modeling and simulation. Through a large scale distributed simulation network, the multiple development teams, who are in the different regions, can share all simulation resources and collaborate for a common development task. When using some techniques to make integrated simulation environment has distributed simulation capabilities, the distributed integrated simulation environment is formed.

\section{B. Distributed Integrated Simulation Research Status}

The aforementioned NPSS select distributed simulation framework for the realization of integrated simulation environment [2]. As shown in the Figure 3 (a), by using of 0$\mathrm{D}$ modules a simulation model of turbofan engine is built, of which the high pressure compressor (HPC) module is not a true simulation model, but using the Common Object Request Broker Architecture (CORBA) standard development of the distributed simulation interface. Through the interface 0-D system model and remote 0-D HPC model (as shown in the Figure 3 (b)) are coupled, achieving simulation zoom.

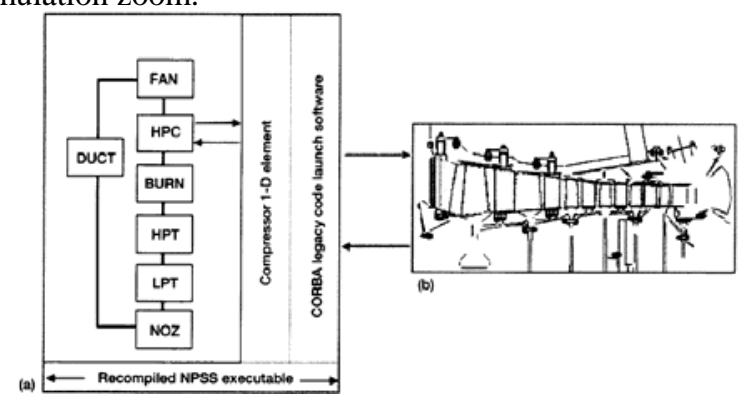

Figure 3. The GSP operating enviroment.

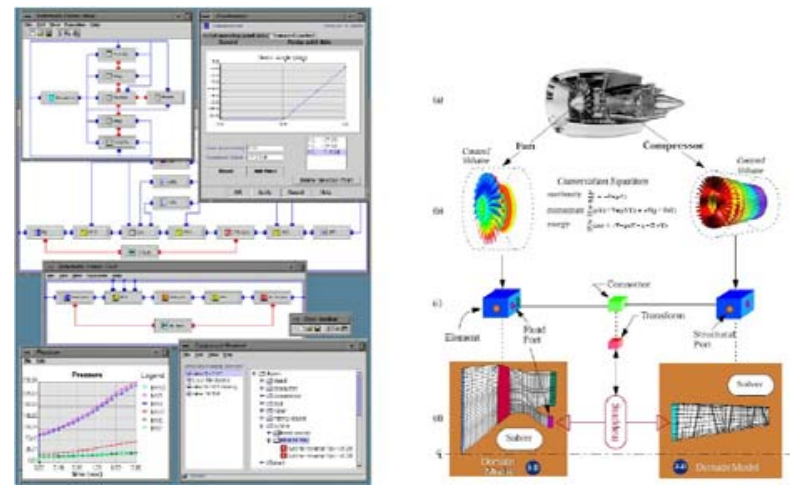

Figure 4. Simulation platform Onyx.

In the NPSS program support, Toledo University and NASA Glenn research center, the use of JAVA language 
develop line with the NPSS standard extensible aircraft engine integrated simulation platform Onyx [14]. The platform has multidisciplinary analysis, simulation zoom, distributed simulation three functions, the distributed simulation is achieved based on the JAVA language remote method invocation function set and CORBA interface. Figure 4 show the Onyx simulation interface and implementation of a example of multidisciplinary analysis and simulation zoom.

Northwestern Polytechnical University Li Zhongqun [15] based on high level architecture technology (HLA) develop aircraft engine full digital soft and hardware integrated simulation platform AESP, builing a high performance distributed simulation environment for the whole or component simulation experiment of the engine.

\section{CONCLUSIONS}

Through summarizing the domestic and foreign gas turbine equipment integrated simulation research can be seen, compared with developed countries, China in the following two aspects of deficiency:

- Lack of based on different development environment, belonging to different areas, with different simulation dimension of the component or subsystem simulation model reuse method research. not forming an expandable, the component or subsystem simulation model has enough reuse capability, and has a uniform interface and operation style of the modular model library.

- Do not form with standardization and open gas turbine distributed integrated simulation platform. Advanced gas turbine equipment integrated simulation platform in general has distributed simulation ability, its reason depends on using distributed simulation framework, can significantly enhance the modeling and simulation of process flexibility, reduce simulation time; and can promote more remote development team for all simulation resources sharing, the realization of simulation model "plug-and-play", improve the development efficiency of gas turbine equipment.

At present our country is in gas turbine research field in the phase of introduction, digestion, absorption to independently developed, there is an urgent need for a general, scalable integrated simulation software, to support the gas turbine equipment general performance matching analysis and parameter evaluation and optimization. From the point of long-term development, China should develop a long-term planning, which is similar to American NPSS plan, based on the distributed simulation software framework, develop a gas turbine equipment integrated simulation software. The software uses standard of distributed simulation interface, to the maximum extent possible to reuse existing autonomy simulation models of component or subsystem.

\section{ACKNOWLEDGMENT}

The authors thank Prof. HongTao Zheng, Prof. WeiLing Xie, Dr. YunPeng Cao, Dr. XiaoYun Zhang, and Dr. WeiYing Wang for valuable discussions about integrated simulation technology for gas turbine equipment. Their detailed and knowledgeable suggestions for improvements greatly improved the quality of the paper.

\section{REFERENCES}

[1] Zhou Xiang, Xie Tingyi, “The development of general integrated simulation platform”, Computer simulation. Vol. 27, Mar. 2010, pp. 295-297.

[2] G. Follen, "Numerical Zooming Between a NPSS Engine System Simulation and a One-Dimensional High Compressor Analysis Code”, NASA TM-2000- 209913, 2000.

[3] Robert L. Bayt, Michael K. Sahm and Charles E. Lents, "Collaborative Engineering in Integrated Aircraft Power System Desing”, ASME DETC2002/ISD- 34439, 2002.

[4] Su Sanmai, “Gas turbine engine simulation software GSP”, The third aircraft Engine reliability academic exchange of China aircraft society, 2005, pp. 181-185.

[5] Xie Zhiwu, Su Ming and Weng Shilie, "Extensible object-oriented simulation models for gas turbine”, Journal of aircraft power. Vol. 14, Apr. 1999, pp. 1-7.

[6] Zhao Wei, "Research of gas turbine modeling simulation integration support system”, Shanghai Jiao Tong University, Doctoral dissertation, 2008, pp. 1-86.

[7] V. Panov, "GASTURBOLIB - SIMULINK LIBRARY FOR GAS TURBINE ENGINE MODELLING”, ASME GT2009-59389, 2009.

[8] Antonio Alejandro Gómez Pérez, "Modelling of a Gas Turbine with Modelica”, ISRN LUTFD2/TFRT-5668-SE, 2001.

[9] K. G. Kyprianidis, A. I. Kalfas, "Dynamic performance investigations of a turbojet engine using a cross-application visual oriented platform”, THE AERONAUTICAL JOURNAL. Vol. 3196, Mar. 2008, pp: 161-169.

[10] Yuan Cao, Xianlong Jin and Guang Meng, "Computational modular model library of gas turbine”, Advances in Engineering Software. Vol. 36, Sep. 2005, pp: 127-134, doi:10.1016/j.advengsoft.2004.07.006.

[11] Liu YongWen, "System modeling and semi-physical simulation based on universal platform and the application in the marine power equipment”, Shanghai Jiao Tong University, Doctoral dissertation, 2002, pp. 1-85.

[12] Bao Wen, Li Weipeng and Chang Juntao, “The distributed parameter closed-loop control simulation system based on the FLUENT/MATLAB port”, JOURNAL OF SYSTEM SIMULATION. Vol. 20, Nov. 2008, pp. 2851-2855.

[13] Qi Xinjie, Guo Yingqing and Wang Haiquan, "Integrated simulation platform of aircraft engine control system”, Science Technology and Engineering, Vol. 9, Oct. 2009, pp. 2685-2691.

[14] J. A. Reed, "Onyx: An Objected-Oriented Framework for Computational Simulation of Gas Turbine Systems”, The University of Toledo, Doctoral dissertation, 1998.

[15] Li Zhongqun, "System simulation and the application study in the aircraft engine simulation platform design", Northwest industry university, Master dissertation, 2004, pp. 22-65. 